\title{
Inhibición del Oscurecimiento con Mucílago de Nopal (Opuntia ficus indica) en el Secado se Plátano Roatán
}

\author{
Laura V. Aquino, Juan Rodríguez, Lilia L. Méndez y Kenia F. Torres \\ Centro Interdisciplinario de Investigación para el Desarrollo Integral Regional, Laboratorio de \\ Tecnología Agroalimentaria CIIDIR-IPN-Unidad Oaxaca, Hornos 1003, Santa Cruz Xoxocotlán, \\ 71230 Oaxaca, Oaxaca-México \\ (e-mail: lauvicka@hotmail.com, jrodrigr@hotmail.com, mendezll@ipn.mx)
}

\begin{abstract}
Resumen
El objetivo de este estudio fue inhibir el oscurecimiento durante el deshidratado de plátano Roatán (Musa cavendish), usando una solución de mucílago de nopal (Opuntia ficus indica) combinado con diferentes concentraciones de acido cítrico y bisulfito de sodio. El tratamiento se aplicó siguiendo un diseño experimental $2^{3}$, siendo los factores y niveles: pre-tratamiento (mucílago-acido cítricobisulfito de sodio) y concentración (alta y baja). Para el secado se usaron cortes transversales de plátano de un espesor de $5 \mathrm{~mm}$; una temperatura de $50{ }^{\circ} \mathrm{C}$ y una velocidad de aire de $2 \mathrm{~ms}^{-1}$. Se midió el color antes y después del secado y se consideró como variable respuesta. La combinación de mucílago con ácido cítrico y bisulfito de sodio a altas concentraciones tuvo un efecto sinérgico que favorece en la disminución del oscurecimiento del plátano durante el secado. El mucílago formó una cubierta protectora en la superficie que proporcionó brillo al material deshidratado.
\end{abstract}

\section{Inhibition the Darkening with Cactus Mucilage (Opuntia ficus indica) during Drying of Banana Roatán}

\begin{abstract}
The object of this study was inhibiting the browning process during the drying of banana Roatán (Musa Cavendish) using a solution of cactus mucilage (Opuntia ficus indica) blend with different concentrations of citric acid and sodium bisulphite. The treatment was applied following an experimental design $2^{3}$, using the following factors and levels: pre-treatment (mucilage, citric acid, sodium bisulphate) and concentration (high and low). For the drying cross sections of banana with thickness of $5 \mathrm{~mm}$, temperature of $50{ }^{\circ} \mathrm{C}$ and air velocity of $2 \mathrm{~ms}^{-1}$ were used. Color before and after drying was determined and was consider as the response variable. The combination of mucilage, citric acid and sodium bisulphite at high concentrations had a synergic effect that diminished the browning of bananas during drying. The mucilage formed an edible coating in the surface that gives shine to the dried material.
\end{abstract}

Keywords: banana Roatán, cactus mucilage, browning, mucilage, pre-treatment 


\section{INTRODUCCION}

En la actualidad los consumidores exigen alimentos preservados lo más parecido a los frescos y que garanticen una larga duración. El secado de alimentos en un método que permite la conservación de Ios alimentos; un alimento deshidratado aceptable debe tener un sabor, color y apariencia comparable con el fruto fresco ó con productos procesados por otros medios, debe reconstituirse fácilmente, retener los valores nutritivos y ser estable durante el almacenamiento (Moranga et al., 2006).

Algunos alimentos requieren de un tratamiento previo al secado, este ayuda a bloquear la acción de los agentes microbianos ó enzimas que pueden alterar las características originales del alimento. Al aplicar tratamientos térmicos se generan tonalidades que van desde un ligero amarillo hasta un intenso café mediante reacciones de Maillard y de caramelización. El uso de inhibidores químicos ha sido el método común para evitar el oscurecimiento; en estudios realizados se ha encontrado que el ácido ascórbico, cisteína, acido cítrico, acido acético, son buenos inhibidores del oscurecimiento enzimático de la pulpa de plátano (Yang y Shuji, 2000). El dióxido de azufre y sulfitos son fuertes inhibidores de polifenoloxidasas y comúnmente usados en alimentos, sin embargo, su uso tiene ventajas y desventajas, se usa en casos donde la aplicación de calor cambia la textura ó la pérdida de sabor, provocando olores y sabores desagradables; y a concentraciones elevadas destruyen algunas propiedades nutritivas del alimento provocando daños en la salud (McEvily et al., 1992).

La demanda para reducir químicos en el proceso de frutos y vegetales, ha orientado a la búsqueda de sustancias naturales que reduzcan el ataque microbiano y la oxidación. El uso de películas comestibles y recubrimientos para la protección y preservación de los alimentos tiene algunas ventajas: es un producto biodegradable y no contamina (Tharanathan, 2003), prolongan la vida de anaquel y protegen al alimento del ataque microbiano (Quintavalla y Vinici, 2002), su capacidad para retardar la humedad, el oxigeno, los aromas, y el transporte de solutos, puede mejorarse con la inclusión de aditivos tales como antioxidantes, antimicrobianos, colorantes, sabores, fortificadores de nutrientes y especias en la formulación de la película (Pranoto et al., 2005).

El mucilago de nopal es un polímero compuesto por polisacáridos semejante a las pectinas, ésta propiedad así como sus características reológicas le dan un potencial como materia prima para la elaboración de películas plásticas comestibles (Arizmendi, 2004). Del Valle et al. (2005), comprobó la eficiencia del una película comestible de mucilago de nopal como recubrimiento en fresas (Fragaria ananassa), observó el incremento de vida útil sin afectar el color y sabor del alimento. Por lo tanto el objetivo de este estudio fue evaluar el efecto del mucilago del nopal con diferentes concentraciones de bisulfito de sodio y ácido cítrico como pre-tratamiento a rodajas de plátano Roatán sometido al secado convectivo con temperatura de $50{ }^{\circ} \mathrm{C}$ y velocidad de aire de $2 \mathrm{~ms}^{-1}$.

\section{METODOLOGIA}

En la comunidad de Ayoquesco de Aldama perteneciente al municipio de Zimatlán de Álvarez Oaxaca, en el mes de noviembre se recolectaron en costales 40 cladodios maduros de de Opuntia ficus indica (1 a 2 años de edad aprox.) y se trasladaron al laboratorio de Tecnologías Agroalimentarias del CIIDIR-IPN Unidad Oaxaca.

Los cladodios se lavaron con un cepillo para eliminar espinas y polvo; se pesaron $2 \mathrm{~kg}$ de cladodios en una balanza Navigator marca Ohaus $(8000 \mathrm{~g} \pm 0.5 \mathrm{~g})$, una vez pesados se les retiró la cutícula con un cuchillo y el parénquima se troceó obteniendo cubos de $9 \mathrm{~cm}^{3}$ aproximadamente. En una parrilla Thermolyne en una olla se calentaron $1500 \mathrm{~mL}$ de agua a temperatura de $94 \pm 2^{\circ} \mathrm{C}$, dentro de la olla los trozos se colocaron en una malla metálica y se escaldaron durante 7 min. Concluido el escalde los trozos se retiraron, se colocaron en un recipiente de plástico y se agregó $2000 \mathrm{~mL}$ de agua destilada (relación 1:1 p/p), dejando macerar por $24 \mathrm{~h}$ en un refrigerador domestico Mabe a una temperatura de $5 \pm 2^{\circ} \mathrm{C}$, después de la maceración se decanto el mucílago y se centrifugo a 628.32 rads $^{-1}$ en una centrifuga marca Dynac modelo 228125 durante $10 \mathrm{~min}$, con este proceso se separó el mucilago de los residuos de nopal. El mucilago extraído se mezcló con acetona (1:2 v/v), el precipitado se lavó con alcohol iso-propílico (Medina et al., 2000) y se obtuvo una goma que se 
secó a $50{ }^{\circ} \mathrm{C}$ en una estufa de vacio marca Shel-Lab modelo 14300, el mucílago seco se almacenó a temperatura ambiente $24 \pm 2^{\circ} \mathrm{C}$ en bolsas de poliuretano selladas al vacio.

Se probaron diluciones de mucilago deshidratado con viscosidades de 10 a $40 \mathrm{mPas}$ medidas en un viscosímetro Brookfiel Synchro-Lectric; se observó que con las diluciones de 20 y 35 mPas hubo mejor adherencia en las rebanadas de plátano, por lo tanto, para viscosidad de $35 \mathrm{mPas}$ se diluyeron $8.5 \mathrm{~g}$ de mucilago deshidratado en $1200 \mathrm{~mL}$ de agua destilada, para obtener $20 \mathrm{mPas}$ se diluyeron 3 gr de mucilago en $1200 \mathrm{~mL}$.

Para evaluar el efecto del mucilago como inhibidor del oscurecimiento durante el secado de plátano, se adquirió en un mercado de la ciudad de Oaxaca plátano Roatán (Musa cavendish spp) con etapa de madurez 7 fruto con tonalidad amarilla y puntos cafés. Del fruto se cortaron rebanadas de $5 \pm 0.6$ $\mathrm{mm}$ de espesor y se sumergieron en una solución de mucilago - acido cítrico y bisulfito de sodio (Tabla 1) por 15 minutos con la finalidad de que la solución cubriera completamente la rebanada.

Tabla 1. Condiciones de pre-tratamiento de plátano roatán

\begin{tabular}{|c|c|c|c|}
\hline Pre-tratamiento & $\begin{array}{c}\text { Ácido cítrico } \\
(\%)\end{array}$ & $\begin{array}{c}\text { Bisulfito de sodio } \\
\text { (ppm) }\end{array}$ & $\begin{array}{c}\text { Mucílago } \\
\text { (mPas) }\end{array}$ \\
\hline 1 & 1 & 500 & 35 \\
\hline 2 & 1 & 500 & 20 \\
\hline 3 & 1 & 200 & 35 \\
\hline 4 & 1 & 200 & 20 \\
\hline 5 & 0.5 & 500 & 35 \\
\hline 6 & 0.5 & 500 & 20 \\
\hline 7 & 0.5 & 200 & 35 \\
\hline 8 & 0.5 & 200 & 20 \\
\hline
\end{tabular}

Las rebanadas tratadas se colocaron en 4 charolas de $25 \times 25$, se seleccionaron 10 testigos a los que se les midieron por triplicados los parámetros de color $L^{*}$ (luminosidad 0=negro y 100=blanco),

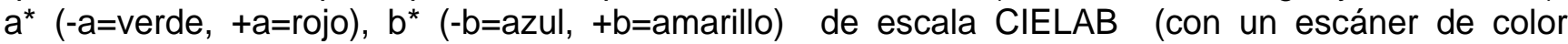
miniescan Hunter Lab MS/B20005. Como variable respuesta se calculó el valor de $\Delta \mathrm{E}$ (diferencia total de color) con la siguiente expresión:

$\Delta \mathrm{E}=\left(\left(\Delta \mathrm{L}^{\star}\right)^{2}+\left(\left(\Delta \mathrm{a}^{\star}\right)^{2}+\left(\left(\Delta \mathrm{b}^{\star}\right)^{2}\right)^{0.5}\right.\right.$

Donde:

$\left(\Delta \mathrm{L}^{*}\right)^{2}=\left(\mathrm{L}^{*} \mathrm{O}^{*} \mathrm{~L}^{2}\right.$

$\left(\Delta \mathrm{a}^{\star}\right)^{2}=\left(\mathrm{a}^{*}{ }_{0}-\mathrm{a}^{\star}\right)^{2}$

$\left(\Delta b^{\star}\right)^{2}=\left(b^{\star}{ }^{*}-b^{\star}\right)^{2}$

Los parámetros $L^{*}, a_{0}{ }_{0}, b^{*}{ }_{0}$ son los valores de las muestras con pre-tratamiento y los valores $L^{*}, a^{*}$, $b *$ son los valores medidos cada 30 minutos durante el secado.

Las charolas con las muestras se secaron en un secador de túnel (Rodríguez et al., 2001) el aire fluyó longitudinalmente en las muestras a una velocidad de $2 \mathrm{~ms}^{-1}$, la temperatura de secado fue de $50{ }^{\circ} \mathrm{C}$, por 5 horas. Se utilizó un diseño de experimentos factorial $2^{3}$, siendo los factores y niveles: pre-tratamiento (mucílago-acido cítrico-bisulfito de sodio) y concentración (alta y baja), obteniendo un total de 8 experimentos, se realizó un análisis de varianza con nivel de significancia de 0.05 , los datos fueron procesados en un paquete estadístico NCSS 2001.

\section{RESULTADOS Y DISCUSION}

En la figura 1 se presentan los valores del parámetro $L^{*}$, se observa la pérdida de luminosidad de las rebanadas de plátano durante el secado, los tratamientos 7 y 8 tuvieron una fuerte disminución de luminosidad, en los tratamientos 1, 2, 5 la pérdida de luminosidad no fue evidente. Los valores de $a^{*}$ 
indicaron una tendencia a la coloración rojiza indicativo de oscurecimiento, los tratamientos 7, 6, 4, 1, 3 tuvieron mayor oscurecimiento, en el 5 y 1 esta coloración no estuvo tan presente (figura 2). Durante el secado a los 60 minutos se presentó el mayor incremento de $a^{\star}$, durante el secado los cambios fueron oscilando el efecto se puede deber a que hay presencia de caramelización, reacciones de Maillard ó enzimáticas, degradación de pigmentos y oxidación de ácido ascórbico.

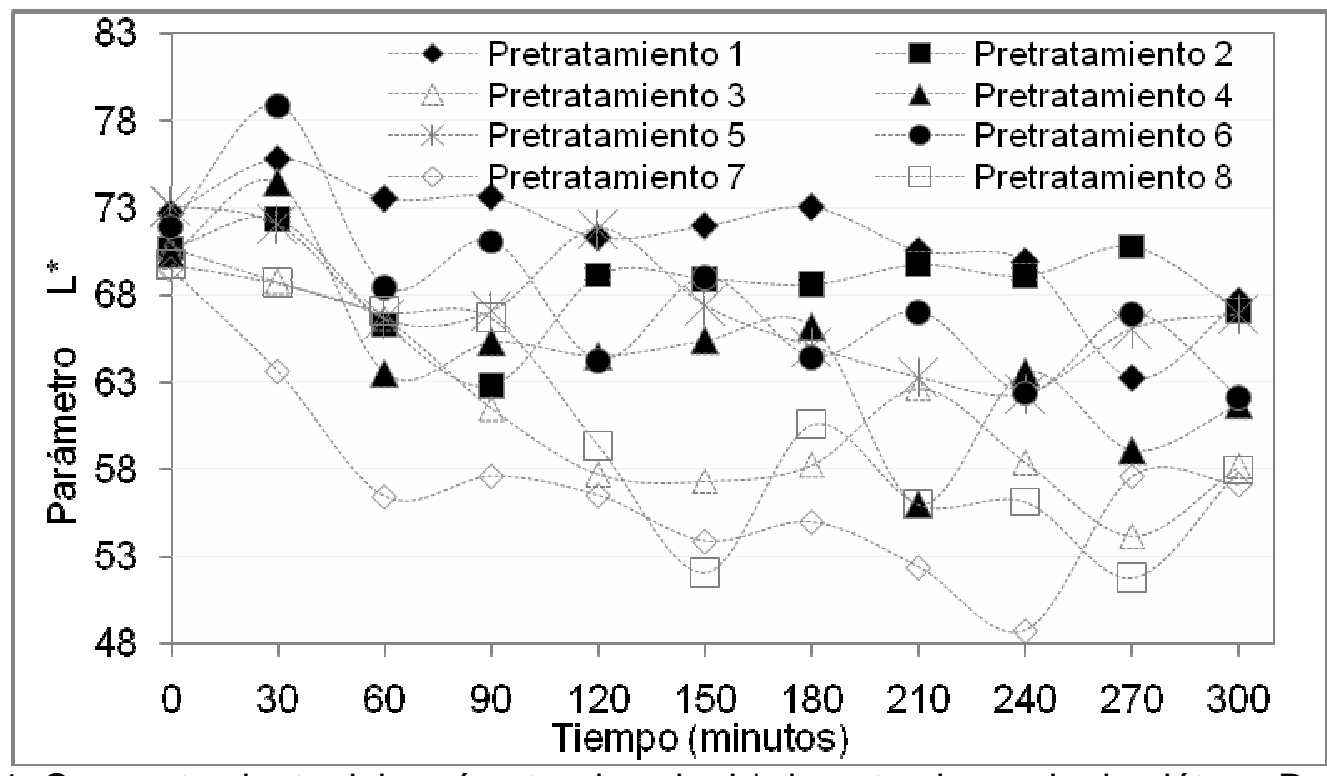

Fig. 1: Comportamiento del parámetro de color $L^{*}$ durante el secado de plátano Roatán.

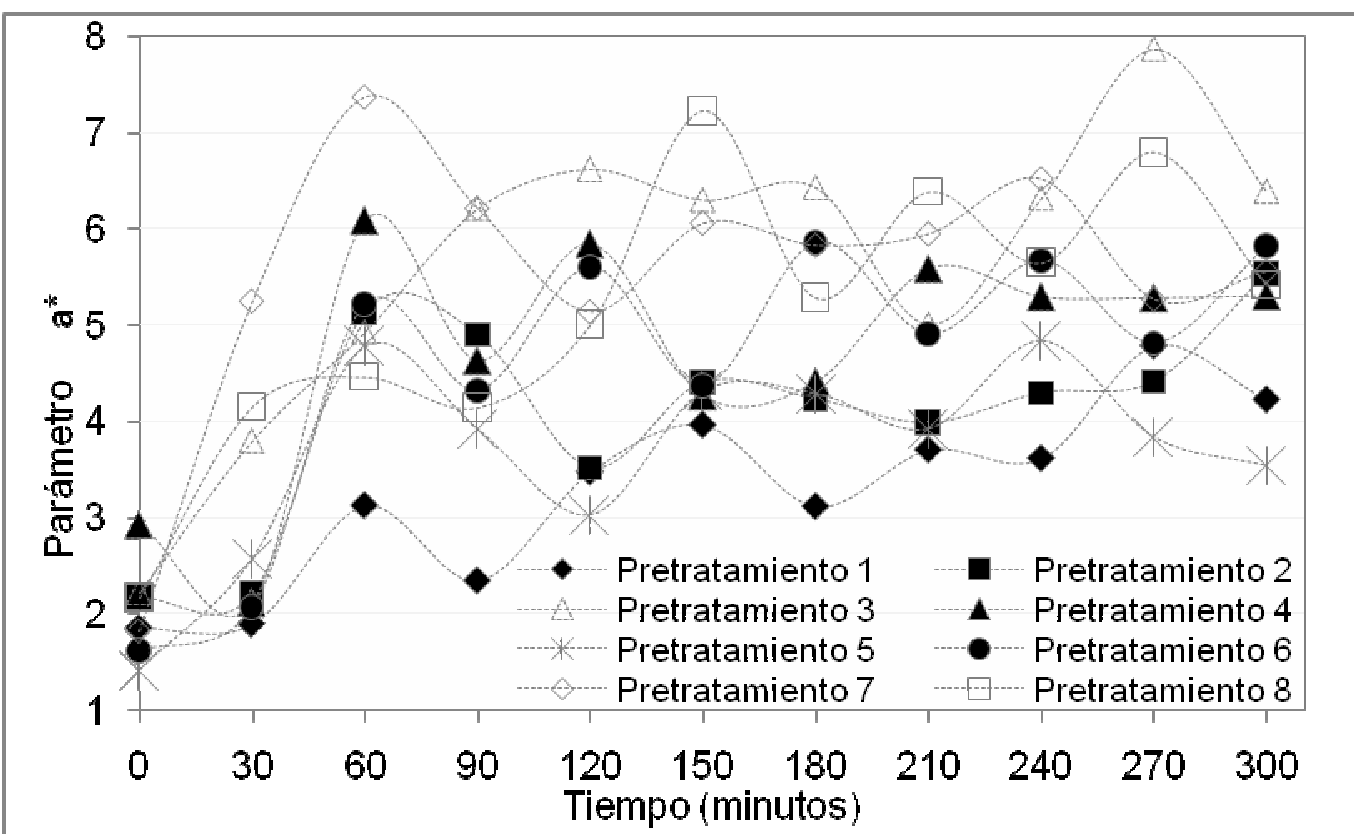

Fig. 2: Comportamiento del parámetro de color a* durante el secado de plátano Roatán.

En la figura 3 se muestra el comportamiento del parámetro b*, los valores positivos indican la tendencia hacia la coloración amarilla, esta coloración se acentuó en los pre tratamientos 2 y 6 , en los pre tratamientos restantes la coloración se mantuvo en un rango de 31 a 28 para el parámetro b*.

En la figura 4 se presenta el comportamiento de la diferencia total de color $\Delta \mathrm{E}$, en las pruebas 1, 5 la presencia del oscurecimiento es menor, al evaluar individualmente los parámetros de color se observó que la alta concentración de bisulfito de sodio y ácido cítrico reducen el oscurecimiento del las rebanadas de plátano, la viscosidad del mucilago solo influye en la luminosidad del material. McEvily et al. (1992) y Saper (1993) explican el efecto sinérgico de la combinación de bisulfito de sodio y el ácido ascórbico como agentes reductores de las o-quinonas formadas por la acción enzimática de los compuestos monofenolicos, formándo de esta forma sulfonatos y sulfatos que 
pueden reducirse nuevamente por la intervención del ácido L-ascórbico, produciendo de esta forma la regeneración de los sulfitos y disminución del oscurecimiento. Los sulfitos y el acido L-ascórbico pueden establecer una rápida interacción con el oxigeno, limitando los eventos oxidativos catalizados por la polifenoloxidasa y peroxidasa.

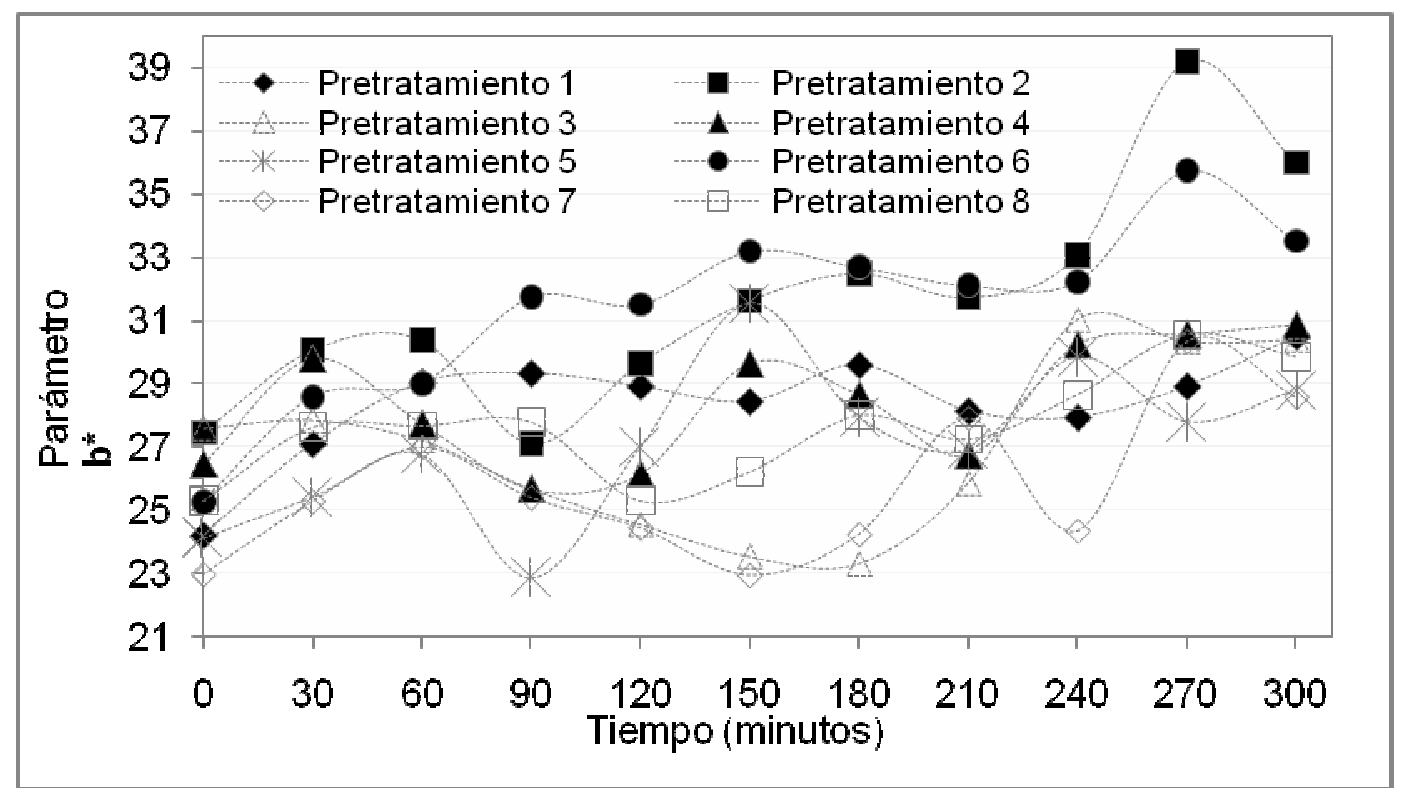

Fig. 3: Comportamiento del parámetro de color b* durante el secado de plátano Roatán.

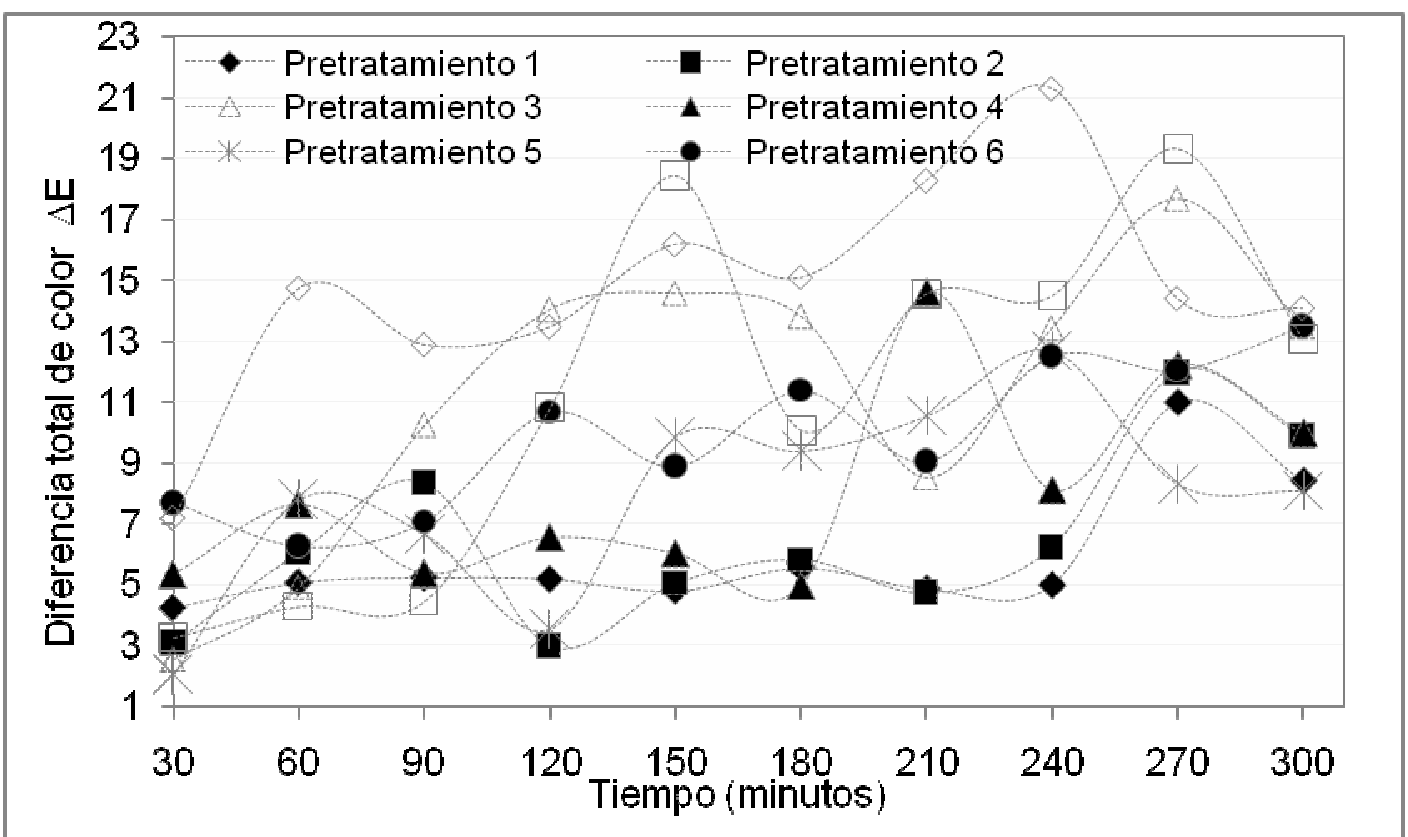

Fig. 4: Comportamiento de $\Delta \mathrm{E}$ durante el secado de plátano Roatán.

Al procesar los resultados en el software NCSS con un alfa de 0.05 se confirmo que a altas concentraciones de bisulfito de sodio se reduce el oscurecimiento, la interacción entre bisulfito y mucilago favorecen a la luminosidad de la muestra, ya que el mucilago forma una barrera brillosa en la superficie de la rebanada. El parámetro significativo fue la concentración de ácido cítrico en los estudios McEvily et al (1992) observaron que bajas concentraciones de ácido cítrico tienen propiedades quelantes y acidulantes que propician un ambiente óptimo para la actividad de las enzimas responsables del oscurecimiento. El análisis estadístico demostró que la inhibición del oscurecimiento de puede obtener por el efecto sinérgico de los tres factores, de manera independiente su capacidad inhibidora es mínima o bien puede provocar una efecto desagradable en la calidad del producto deshidratado, como lo menciona Pranoto et al. (2005) y Millan et al., (2001). 


\section{CONCLUSIONES}

En este estudio el uso de mucilago como cubierta protectora proporciono al material brillo, lo cual se observo en la cinética del parámetro de color $L^{*}$, la concentración de 500 ppm de bisulfito de sodio es la adecuada para la reducción del oscurecimiento, la variación en la concentración de ácido cítrico como agente inhibidor es un factor que influye en el oscurecimiento del material.

Para la reducción del oscurecimiento en rebanadas de plátano Roatán secadas a $50{ }^{\circ} \mathrm{C}$ se requirió de un pre-tratamiento con una solución concentrada de mucilago de nopal (35 mPas), una concentración de bisulfito de sodio (500 ppm) y de acido cítrico (1\%).

Al evaluar el efecto sinérgico de los tres factores del pre-pre-tratamiento se comprobó que el mucilago de nopal combinado con antioxidantes es una buena alternativa para disminución del oscurecimiento en el secado de plátano Roatán, además de que forma en el material una cubierta protectora puede conservar la calidad del material deshidratado durante el almacenamiento .

\section{REFERENCIAS}

Arizmendi C. D.; Optimización de dos compuestos plastificantes (Glicerol y Polietilenglicol) en la elaboración de una película comestible obtenida a partir del mucílago de nopal de la especie Opuntia tomentosa "Salm-Dyck", http://www.pncta.com.mx/pages/pncta_investigaciones_04h.asp?page=04e12, (2004).

Del Valle V.P., M.A. Hernández y M.J. Guarda; Development of cactus mucilage edible coating (Opuntia ficus indica) and its application to extend strawberry (Fragaria ananassa) shelf life. Food Chemistry: 91(4), 751-756 (2005).

McEvily A.J., R. Iyengr y W.S. Otwell; Inhibition of enzymatic browning in foods and beverages Critical, Reviews in Food Scice and Nutrition: 32, 253-273 (1992).

Medina T.L, D.L.F.E. Brito, S.B. Torrestiana y S. Alonso; Mechanical properties of gels formed by mixtures of mucilage gum (Opuntia ficus indica) and carrageenans, Carbohydrates polymers: 52(2), 143-150 (2003).

Millan T., R. Felix y T.V. Roa; Uso de la metodología de superficie de respuesta en la evaluación del pardeamiento en cambur procesado por impregnación al vacio. INC: 26(7), 290-295 (2001).

Moranga G., N. Martínez-Navarrete, y A. Chiralt; Compositional changes of strawberry due to dehydratation cold storage and freezing thawing processes, Journal of Food Processing and Preservation: 30(4), 458-474 (2006).

Pranoto Y., V.M. Salokhe y S.K. Rakshit; Physical and antibacterial properties of aliginate based edible film incorporated with garlic oil, Food Research International: 38 (3), 267-272 (2005).

Quintavalla S. y L. Vinici; Antimicrobial food packaging in meta industry. Meat Science: 62, 373-380 (2002).

Rodríguez R.J., L.L Méndez, A.C. Martínez y N.F. Diego; A closed loop tunnel for drying kinetics, Research Inter-American dying conference, Boca del Rio Veracruz, México (2001).

Saper G.M.; Browning of foods: control by sulfites, antioxidants and other means. Food Technology: 10, 75-84 (1993).

Tharanathan R.; Biodegradable films and composite coating: past, present, and future. Critical Review in food, Science and Tecnology,:14, 71-78 (2003).

Yang P.C. y F. Shuji; Purification and characterization of polyphenol oxidase from banana (Musa sapientum L.), Journal of Agricultural and Food Chemistry: 48(7), 2732-2735 (2000). 